\title{
A Person with Factitious Disorder Presenting with Acute Stroke-Like Symptoms and Receiving Thrombolytic Therapy Twice
}

\author{
Samir R Belagaje*, Jordan Bonomo, Christopher White, Brett Kissela, Dawn Kleindorfer, Robert Neel and Joseph Broderick \\ Grady Memorial Hospital, Atlanta, GA 30303, United States
}

\section{Introduction}

One of the challenges in acute stroke management is accurately differentiating between actual ischemic events and other conditions that mimic stroke. In a cohort of 821 consecutive patients admitted to an acute stroke unit, $13 \%$ were incorrectly diagnosed as stroke [1]. In another cohort of 411 patients, it was estimated that $19 \%$ of patients presenting to the emergency department with stroke-like symptoms ultimately have other diagnoses such as a postictal state, metabolic disturbances, and systemic infections [2]. Regardless of the actual percentage, it is clear that not all cases of acute stroke-like symptoms are true strokes and other mimics should be considered. One such mimic is factitious disorder.

Factitious disorder is a psychiatric condition in which afflicted individuals exaggerate symptoms and even endorse medical illness, or psychological trauma in order to draw attention or sympathy to themselves. The DSM-IV criteria for this disorder are: 1) Intentional production or feigning of physical or psychological signs or symptoms; 2) The motivation for the behavior is to assume the sick role; and 3) External incentives for the behavior such as economic gain or avoiding legal responsibility as seen in malingering, are absent [3].

Here, we present a case of a patient who was diagnosed with factitious disorder after he presented to two different institutions with acute stroke-like symptoms and received tissue plasminogen activator (t-PA) twice within the span of 3 weeks.

\section{Case Report}

A 53 year old white male presented to a local ED with acute onset of right face, arm and leg weakness. He stated he was a retired military trauma surgeon who had buried his son two days prior whom he had lost in recent military action.

As per the hospital protocol, the ED notified the Stroke Team who evaluated the patient in person and rated the severity of his symptoms with a NIH Stroke Scale (NIHSS) score of 10. As he fulfilled the other inclusion/exclusion criteria, thrombolytic therapy with IV t-PA was started 2.8 hours after symptom onset. The potential risks of such therapy were discussed with the patient prior to initiation and he did not object to the treatment.

The next day, with his weakness improving, the patient's NIHSS was 2. The brain MRI did not reveal any evidence for acute stroke. During the hospital course, the patient provided additional information that gave health care providers pause. For example, he stated his ex-wife had called the day after his son's burial to inquire into the death benefits provided by the government. Based on the negative MRI and some of his statements, the health care team inquired further into his history. All inquiries were carried out in a manner compliant with HIPAA laws as well as the ethical code of the institution. It became apparent that he was not a retired military trauma surgeon, and that he did not have a son who had served or died recently in the military.

After being discharged, the patient returned three weeks later at another institution in a different state with a similar presentation of acute onset of right face, arm and leg weakness. This time, he stated he was a psychologist who helped lawyers select juries and was in the area to attend his wife's funeral. His NIHSS score was unknown at time of presentation. He received t-PA for acute stroke in the emergency department and was transferred to our institution for further management. The facility to which he was transferred is the tertiary referral hospital for our regional stroke team. Given the similarities between the two cases, the treating physicians were able to identify the patient.

He tolerated the second t-PA dose well but developed a minor groin hematoma where a central line had been placed at the outside hospital. A psychiatry consult was obtained as the team considered factitious disorder after further imaging did not reveal evidence for a stroke. The patient was subsequently transferred to inpatient psychiatry on an involuntary 72 hour hold for further management and evaluation. The teams were concerned that the patient had endangered himself by receiving t-PA twice in a matter of three weeks. The teams were also concerned by the extent and nature of the patient's inaccurate stories suggesting that this was not a standard conversion disorder. When confronted that he did not have a stroke during either presentation, he admitted that the symptoms were fabricated and his weakness quickly resolved.

During his inpatient stay, the psychiatry team diagnosed him with two Axis I disorders: 1) Bipolar disorder, and 2) Factitious disorder. He was started on Depakote and Risperdal for his grandiose ideas and participated in group therapy sessions. He was subsequently discharged after his symptoms appeared to be under better control.

\section{Discussion}

Although the literature contains case reports for factitious disorder imitating acute stroke, the actual incidence rates are not known. Furthermore, this is the first report of which we are aware where a patient with factitious disorder was treated twice with thrombolytics. Patients with this disorder are difficult to diagnose and as seen in this case, although there was some initial hesitancy, the patient was ultimately treated with t-PA. The limited time window for thrombolytic therapy in acute stroke and the reliance on clinical exam contribute to the difficulty with diagnosis. Definitive diagnostic imaging tests such as MRI are time consuming and not routinely ordered in the emergency department for acute stroke care. Consequently, care givers tend towards unknowingly treating non-strokes with thrombolytics.

*Corresponding author: Samir R Belagaje, Faculty Office Building \#375, Grady Memorial Hospital, 80 Jesse Hill Jr Dr. SE, Atlanta, GA 30303, United States, E-mail: samirb315@yahoo.com

Received March 15, 2012; Accepted April 23, 2012; Published May 02, 2012

Citation: Belagaje SR, Bonomo J, White C, Kissela B, Kleindorfer D, et al. (2012) A Person with Factitious Disorder Presenting with Acute Stroke-Like Symptoms and Receiving Thrombolytic Therapy Twice. J Clin Case Rep 2:138. doi:10.4172/21657920.1000138

Copyright: (c) 2012 Belagaje SR, et al. This is an open-access article distributed under the terms of the Creative Commons Attribution License, which permits unrestricted use, distribution, and reproduction in any medium, provided the original author and source are credited. 
Patients with this disorder are difficult to diagnose and it is made more difficult when the symptoms mimic an acute stroke. In most cases, history and exam findings can help guide diagnosis. Age is one such factor. In a study of 669 consecutive patients admitted to a hospital with the diagnosis of stroke, $21 \%$ of patients under the age of 50 were ultimately diagnosed with a stroke mimic compared to $3 \%$ of patients over the age of 50 having a stroke mimic [4]. In a case series of 4 patients with factitious disorder presenting with acute stroke-like symptoms, Hemphill and Chung [5] found that the absence of facial involvement but arm and leg hemparesis and fluctuating weakness were signals that should raise possibility of inorganic etiology. However, in this case, the patient did have facial involvement and was consistent with his weakness on repeated testing by several different members of the stroke team, making the diagnosis difficult. Similar to other patients presented in case reports $[5,6]$, when confronted in a professional and respectful manner, the patient admitted that he did not have a stroke and his symptoms quickly resolved.

This case provides different perspectives on the concept of a medication with dangerous complications, such as t-PA. The initial NINDS trial of t-PA for the treatment of acute stroke demonstrated a $6 \%$ risk of symptomatic hemorrhage and no change in mortality compared to placebo [7]. However, this was in patients with actual ischemic strokes. In the GUSTO-1 trial of patients treated with thrombolytics for non-stroke related reasons, the risk of severe bleeding was 1.2\% [8]. In another study of emergency department misdiagnoses in patients treated with t-PA for acute ischemic stroke, none of the non-stroke patients who received t-PA developed hemorrhagic complications [9]. However, it should be mentioned that the study was not designed to detect hemorrhagic complications and given the small number of mimics in the study, safety data is difficult to interpret.

Although the patient in our case was not harmed by the medication, he did experience mild side effects which had the potential to become clinically significant. During his initial treatment, he developed a mild effusion in his right knee from the reported fall at his son's gravesite and during his second treatment, he developed a groin hematoma. A hemarthrosis or larger hematoma would have been severe complications. The risks of such side effects were explained to the patient prior to his initial treatment, and he agreed to the therapy. The patient had demonstrated an ability to endanger himself as repeated treatments may have caused more severe complications.

One characteristic of factitious disorder is "doctor-shopping" by seeking medical care at different institutions. One of the incentives for this behavior is to seek care from health care providers not familiar with their history and therefore more gullible to their signs and symptoms. The drawback of such behavior is that the diagnosis of factitious disorder is delayed and the patients put themselves in danger. One solution to counteract this behavior is if a single care team would be familiar with the patients and treat them across different institutions.

In this way, the case also illustrates the unintended benefit of a large multicenter stroke team. Our team, the Greater Cincinnati/Northern Kentucky Stroke Team evaluates and treats acute stroke patients at 18 different hospitals in a tri-state area. The team also receives phone consults from several other hospitals in the area. Our team communicates their experiences with various stroke cases to each other for back-up coverage, educational, and research purposes.

Using our routine practices, several physicians on the team who were familiar with the first presentation became concerned upon hearing about the second presentation due to their remarkable similarities and investigated further. At the time of hearing the second presentation, the patient had already received t-PA but the physicians involved in his care were unfamiliar with his first presentation. Although the patient did receive t-PA twice, it is important to highlight that the emergency room physicians who made the decision to give him thrombolytics during his second presentation were unfamiliar with his prior presentation. Only after his transfer and involvement of stroke team members, the patient's identity and background came to light and his psychiatric diagnoses were made quickly thereafter and he was started on appropriate treatment.

In summary, we present the case of a patient who had acute episodes of stroke-like symptoms treated twice with thrombolytic therapy, and was ultimately diagnosed with factitious disorder. Although it is rarely reported in the literature, it is likely that other physicians involved in acute stroke care will see patients with factitious disorder presenting similarly.

\section{References}

1. Norris JW, Hachinski VC (1982) Misdiagnosis of stroke. The Lancet 319: 328 331.

2. Libman RB, Wirkowski E, Alvir J, Rao TH (1995) Conditions that mimic stroke in the emergency department. Implications for acute stroke trials. Arch Neuro 52: $1119-1122$

3. (1994) Diagnostic and Statistical Manual of Mental Disorders, 4th Edition Washington, DC, American Psychiatric Association, 471-472.

4. Vroomen PC, Buddingh MK, Luijckx GJ, De Keyser J (2008) The incidence of stroke mimics among stroke department admissions in relation to age group. $J$ Stroke Cerebrovasc Dis 17: 418-422.

5. Hemphil JC 3rd, Chung SS (1999) Factitious stroke presenting for acute treatment. J Stroke Cerebrovasc Dis 8: 88 - 90.

6. Tobiano PS, Wang HE, McCausland JB, Hammer MD (2006) A case of conversion disorder presenting as a severe acute stroke. J Emerg Med 30 283-286.

7. (1995) Tissue plasminogen activator for acute ischemic stroke. The Nationa Institute of Neurological Disorders and Stroke rt-PA Stroke Study Group. N Engl J Med 333: 1581-1587.

8. Berkowitz SD, Granger CB, Pieper KS, Lee KL, Gore JM, et al. (1997) Incidence and predictors of bleeding after contemporary thrombolytic therapy for myocardial infarction. The Global Utilization of Streptokinase and Tissue Plasminogen activator for Occluded coronary arteries (GUSTO) I Investigators. Circulation 95: 2508-2516.

9. Scott PA, Silbergleit R (2003) Misdiagnosis of Stroke in tissue plasminogen activator - treated patients: characteristics and outcomes. Ann Emerg Med 42: 611-618. 